\title{
Three-dimensional electron lattice
}

\section{At low temperatures and in strong magnetic fields, electrons (in a semi-conductor) can indeed be made to crystallize into a lattice, confirming a prediction of Wigner half a century ago.}

THE idea that electrons may form into a crystal lattice, originally due to Eugene Wigner, has finally been confirmed after exactly half a century. The delay does not imply indifference. Since C.C. Grimes and G. Adams demonstrated in 1977 that electrons at the surface of liquid helium will form a two-dimensional lattice of the Wigner type, there have been several claims of evidence for three-dimensional electron lattices in materials as different as InSb and the ternary alloy $\mathrm{Hg}_{1-x} \mathrm{Cd}_{x} \mathrm{Te}$. But only now does it seem to be accepted that T.F. Rosenbaum and S.B. Field of the University of Chicago, working with D.A. Nelson (Honeywell, Lexington) and P.B. Littlewood (AT\&T Bell Laboratories) have unambiguously demonstrated a threedimensional electron lattice in $(\mathrm{Hg}, \mathrm{Cd}) \mathrm{Te}$ with $x$ at about 0.24 (Phys. Rev. Lett. 54, $241 ; 1985)$.

The curious sensation engendered by the notion of an electron lattice is that it seems a contradiction of quantum mechanics. If, as we are always being told, electrons are not localized entities but, at the very least, smudges and perhaps even standing waves, how can they be localized at the vertices of a lattice? The answer is that what matters is only the free energy of the system. The uncertainty principle implies that localization entails a penalty (increased kinetic energy) that may be outweighed by the reduction of repulsion energy stemming from regular arrangement on a lattice.

Naturally, the whole idea would be a more direct assault on more elementary principles were it not taken for granted that an electron lattice can form only if embedded in a neutralizing sea of positive electric charge, such as that provided by a solid crystal lattice from which the electrons have been detached. This is part of the reason why the search for electron lattices has concentrated on semiconductors. The best candidates are materials with only a small concentration of charge carriers of high mobility (or small effective mass compared with that of the electron) which also have a large dielectric constant. Whence $\mathrm{Hg}_{1-x} \mathrm{Cd}_{x} \mathrm{Te}$.

The other variable that matters is an external magnetic field, the effect of which is to assist the localization of electrons, at least in a plane perpendicular to the field. (Wave packets are elongated in the field direction.) What Rosenbaum and his associates have done is to follow the process of crystallization of electrons into a lattice at low temperatures (between $1 \mathrm{~K}$ and $10 \mathrm{mK}$ ) under the influence of an increasing field. Their decisive advantage over earlier workers seems to be the homogeneity of their samples and the low temperatures they have achieved.

Recognizing that the crystallization of electrons is not perhaps as simple as might be expected, but depends on a measurement of the resistivity and Hall resistivity of the sample, the standard means of estimating the mobility and density of charged carriers in a conductor. (Nothing more magical than Faraday's laws of electromagnetism is involved; electric charges moving perpendicular to a magnetic field are also dragged sideways, creating a voltage perpendicular to both the field and the carrier current.)

In these terms, the phenomenon of crystallization is dramatic enough. Rosenbaum et $a l$. find that the Hall resistivity of their $(\mathrm{Hg}, \mathrm{Cd}) \mathrm{Te}$ alloy is only small when the external magnetic field is zero and that it remains virtually constant with increasing field until some critical value of the field (about 6,000 Oe at $10 \mathrm{mK}$ ) is reached, after which the resistivity increases rapidly (and linearly) with increasing field.

The critical field at which the supposed crystallization occurs is, as it should be, an increasing (again linear) function of the temperature. The inference, that the sudden loss of mobility is a mark of the onset of electron crystallization is possible largely because of the low temperatures at which the observations have been made, for only then can other explanations be excluded; the trapping of individual charge carriers by donor atoms is the most obvious of these.

For what it is worth, the measurements agree well with calculations, notably those of W.G. Kleppman and R.J. Elliott ( $J$. Phys. C. 8, 2729; 1975) who set out by representing localized electrons as gaussian wave packets whose dispersion is different (and greater) in the direction of the magnetic field than in the two transverse directions. The calculations show that circumstances may indeed arise in which the repulsive Coulomb energy of electrons in a regular array exceeds the increase of kinetic energy occasioned by localization. Kleppman and Elliott point out that the physical explanation is really quite simple; the Coulomb energy is proportional to the inverse of the electron separation but the kinetic energy to the square of that inverse, so that the former must dominate the latter if the separation is large (or the density of carriers low). The calculations show that the exchange energy between a localized array of electrons is negligible (if the density is low enough), so that the exclusion principle is immaterial, which is something of a surprise.

One accompaniment of this argument which in part accounts for present interest in electron lattices is that a crystalline array of electrons should also be spin-polarized, with the electron spins aligned along the magnetic field. Rosenbaum et al. have indeed accumulated evidence for such an alignment from the irregularities apparent in the increase of resistivity (not the Hall resistivity) with increasing field strength. Alignment, in their case, occurs at a lower field strength than crystallization. So would it not be possible to demonstrate the crystallization of the electrons directly, perhaps by neutron diffraction? Unfortunately, Kleppman and Elliott concluded some time ago (J. Phys. C. 8, 2737; 1975) that the prospects are not bright.

Two sets of issues will now come to the fore. First, the calculation of the phase transition from free to frozen electrons needs attention. So far, people have simply made calculations of the energy of the two phases at zero temperature, so that there is nothing to be said about the dynamics of the transition. The problems clearly, however, have a great deal in common with those arising in the calculation of electron disorder in superconductivity and of the chargedensity waves such as are responsible for the electronic behaviour of, for example, polyacetylene and the other materials which are better candidates as organic superconductors.

The practical implications of this development are less easily foreseen. Obviously, it is good that there is now a physical explanation of a phenomenon that may affect the electronic behaviour of an important set of materials. Whether it will be possible to exploit the fact that the lattice electrons are spin polarized in the same direction remains to be seen, but the low temperature at which crystallization unambiguously occurs is an impediment or a challenge to people's ingenuity. Meanwhile, the demonstration of this phenomenon is yet another proof of the astonishing fertility of Eugene Wigner's contributions, at the outset of his career, to quantum mechanics in particular and to physics in general.
John Maddox 